\title{
Vesicoureteral reflux: we have yet to complete our learning
}

\author{
Hans G. Pohl ${ }^{1,2}$ • J. Peter de Winter ${ }^{3,4}$ - Gregorio P. Milani ${ }^{5,6}$ \\ Published online: 19 February 2021 \\ (C) The Author(s), under exclusive licence to Springer-Verlag GmbH, DE part of Springer Nature 2021
}

Since the application of continuous antibiotic prophylaxis (CAP) for the management of vesicoureteral reflux (VUR), the adage "less is more" has been a guiding principle [1]. The diagnosis of VUR during the prenatal period or following recurrent febrile urinary tract infections and associated with acquired renal cortical scarring is relatively not very common. VUR increasingly became a disease that children lived with, rather than suffered from, notably once it was recognized that VUR spontaneously resolved and CAP prevented UTIs during observation, in most [1]. However, not all children fare well on CAP, and the rising incidence of bacterial resistance requires that we challenge the current paradigm. Studies have called into question CAP's wholesale application, identifying children at the most significant risk for recurrent UTIs and its consequences as those who benefit most from CAP [1]. Variables increasing one's risk for recurrent UTI include age $<6$ months, presence of foreskin, female gender, dilating VUR (i.e., grades 3-5), renal cortical scarring, and bladderbowel dysfunction (BBD) [2]. Whether or not CAP is applied, those who fail no surgical management meet criteria for surgical correction of VUR. There again, risk factors matter in ensuring the successful correction of VUR as the primary

Hans G. Pohl

hpohl@childrensnational.org

1 Division of Urology, Children's National Hospital, Washington, DC, USA

2 George Washington University School of Medicine and Health Sciences, Washington, DC, USA

3 Department of Pediatrics, Spaarne Gasthuis, Hoofddorp/ Haarlem, Netherlands

4 Department of Development and Regeneration, KU Leuven, Leuven, Belgium

5 Pediatric Unit, Fondazione IRCCS Ca' Granda Ospedale Maggiore Policlinico, Milan, Italy

6 Department of Clinical Sciences and Community Health, Università degli Studi di Milano, Milan, Italy endpoint. Generally speaking, endoscopic management has been recommended for lower grades of VUR in uncomplicated patients. In comparison, open correction is the mainstay treatment for higher grades of VUR and patients with BBD.

Mina-Riascos and coworkers [3] should be commended for their effort in filling a void in the literature to understand better the comparative effectiveness of endoscopic versus open correction of vesicoureteral reflux, specifically for high-grade VUR. In their network meta-analysis, including nine studies and more than 1400 renal units, the authors found no differences in UTIs for patients undergoing endoscopic management than vesicoureteral reimplantation. The first AUA Guidelines (1997) established that surgical correction of VUR could be attained in $99.1 \%$ of grade 1 , $99.1 \%$ of grade $2,98.3 \%$ of grade 3, $98.5 \%$ of grade 4 , and $80.7 \%$ of grade 5 , endoscopic management not yet being available [4]. These data establish the gold standard against which other methods need to be compared. Moreover, the authors of the guidelines found that while surgical correction did not wholly eradicate UTIs, the number of febrile UTIs was significantly diminished following surgical correction. The reduction in the incidence of febrile UTIs after ureteral reimplantation suggests that overall the children remained at risk for UTI. Recall that these initial guidelines included many children who presented with UTI.

The authors of the 2010 guidelines (revised in 2017) found that the presence of BBD did not modify the outcome of open surgical correction (97\% rate of correcting VUR with and without BBD) but did impact the success rate following endoscopic correction (89\% success rate without BBD and 50\% success rate with BBD) [5]. The revised guidelines summarized the observations made by many that the presence of BBD in children with VUR impacted care significantly. At each grade of VUR, the presence of BBD was associated with a higher likelihood of recurrent UTI and a lower likelihood of resolution of VUR. The risk of recurrent UTI among children on prophylaxis was $12 \%$ in the absence of concomitant BBD and $44 \%$ with BBD. Recurrent UTIs before surgical correction correlated to the risk for recurrent UTIs after surgery: The 
incidence of postsurgical UTI was $4.8 \%$ among those without documented BBD and $22.6 \%$ among those with BBD.

While it is promising to see that endoscopic correction might be successfully performed for high-grade VUR, without controlling for differences in presentation among the studies and considering the multivariable nature of risk for recurrent UTI, VUR, BBD, and renal scarring, it is still difficult to adopt the endoscopic treatment approach in day-to-day practice. The absence of patient selection criteria related to the mentioned factors remains the major limitation to this work, as acknowledged by the authors, and raises a challenge to those of us dedicated to pediatric urology. Future studies should seek to evaluate precise subgroups of patients with very defined risk profiles, and multi-institutional protocols will be required to expedite enrollment.

Abbreviations UTI, Urinary tract infections; CAP, Continuous antibiotic prophylaxis; VUR, Vesicoureteral reflux; BBD, Bladder-bowel dysfunction

\section{References}

1. Buettcher M, Trueck J, Niederer-Loher A, Heininger U, Agyeman P, Asner S, Berger C, Bielicki J, Kahlert C, Kottanattu L, Meyer
Sauteur PM, Paioni P, Posfay-Barbe K, Relly C, Ritz N, Zimmermann P, Zucol F, Gobet R, Shavit S, Rudin C, Laube G, von Vigier R, Neuhaus TJ (2020) Swiss consensus recommendations on urinary tract infections in children. Eur J Pediatr. https://doi.org/ 10.1007/s00431-020-03714-4

2. Khan A, Jhaveri R, Seed PC, Arshad M (2019) Update on associated risk factors, diagnosis, and management of recurrent urinary tract infections in children. J Pediatric Infect Dis Soc 8:152-159. https:// doi.org/10.1093/jpids/piy065

3. Mina-Riascos SH, Fernández N, García-Perdomo HA (2021) Effectiveness and risks of endoscopic management compared to vesicoureteral reimplantation in patients with high-grade vesicoureteral reflux: systematic review and network meta-analysis. Eur J Pediatr. https://doi.org/10.1007/s00431-021-03948-w

4. Skoog SJ, Peters CA, Arant BS Jr, Copp HL, Elder JS, Hudson RG, Khoury AE, Lorenzo AJ, Pohl HG, Shapiro E, Snodgrass WT, Diaz M (2010) Pediatric vesicoureteral reflux guidelines panel summary report: clinical practice guidelines for screening siblings of children with vesicoureteral reflux and neonates/infants with prenatal hydronephrosis. J Urol. 184:1145-1151. https://doi.org/10.1016/j. juro.2010.05.066

5. Peters CA, Skoog SJ, Arant BS Jr, Copp HL, Elder JS, Hudson RG, Khoury AE, Lorenzo AJ, Pohl HG, Shapiro E, Snodgrass WT, Diaz M (2010) Summary of the AUA guideline on management of primary vesicoureteral reflux in children. J Urol. 184:1134-1144. https://doi.org/10.1016/j.juro.2010.05.065

Publisher's note Springer Nature remains neutral with regard to jurisdictional claims in published maps and institutional affiliations. 